\title{
PROKLA-Redaktion
}

\section{Editorial: Energiekämpfe: Interessen, Kräfteverhältnisse und Perspektiven}

Das auf der Verbrennung der fossilen Energieträger Öl, Kohle und Gas beruhende Energiesystem befindet sich in einer Krise. Zwar dürften die fossilen Vorkommen noch eine Weile reichen - das gilt umso mehr, als sowohl beim Öl als auch beim Gas zunehmend auf unkonventionelle Ressourcen (Teersand, Schieferöl- und -gas) zurückgegriffen wird. Allerdings wird die Verbrennung von Öl und Kohle aus klima-, in Ländern wie China auch aus gesundheitspolitischen Gründen zunehmend kritisiert. Am Abbau und an der energetischen Nutzung der besonders klimaschädlichen Braunkohle entzündet sich derzeit in einigen Ländern eine Protestbewegung, die sich auf lokale Anti-Kohle-Initiativen ebenso wie auf zentrale Veranstaltungen in Gestalt von Protest-Camps oder direkte Interventionen an Tagebauen stützt. Erdgas ist zwar im Vergleich zu Öl und Kohle weniger umweltschädlich, jedoch wird die Gasversorgung in Europa von den jüngsten geopolitischen Spannungen zwischen Russland und der EU bzw. der NATO überlagert.

Außer durch Ressourcenkonflikte und die Überlastung der Senken - also jener Ökosysteme, die die Emissionen aus der Verbrennung fossiler Energieträger absorbieren und damit eine wichtige klimatische Regulierungsfunktion ausüben - ist das fossilistische Energiesystem auch vonseiten der erneuerbaren Energien unter Druck geraten. Deren Nutzung befindet sich weltweit im Aufschwung. In Deutschland ist ihr Anteil am gesamten Endenergieverbrauch zwischen 2000 und 2013 auf 12,4 Prozent gestiegen und hat sich somit verdreifacht; was den Strom angeht, wird mittlerweile mehr als ein Viertel des Bedarfs von erneuerbaren Energien gedeckt (Umweltbundesamt 2015: 34f.). Großkonzerne wie RWE oder E.ON, die über Jahrzehnte hinweg mit der Energieversorgung aus fossilen und atomaren Quellen viel Geld verdient haben, befinden sich in einer Existenzkrise, der sie mit einem grundlegenden Umbau ihrer Unternehmensstrukturen zu begegnen versuchen.

Der Übergang von einem fossilistischen zu einem auf erneuerbaren Energieträgern beruhenden System, dessen Möglichkeit sich in diesen Entwicklungen andeutet, ist jedoch keineswegs konfliktfrei geschweige denn ausgemacht. In Deutschland wurde die Dynamik des dezentralen Ausbaus der Stromerzeugung mittels Solar- und Windkraft durch die Novellierungen des Erneuerbare-Energien-Gesetzes (EEG) in den Jahren 2014 und 2016 gebremst. Profiteure der EEG-Novelle sind eben jene Großkonzerne der Energieversorgung, die mit der Krise des Fossilismus selbst in eine existenzbedrohende Lage geraten sind. Ihnen soll etwa durch die Förderung zentraler Großstrukturen der Stromerzeugung - vor allem in Gestalt von offshoreWindparks, die für die bisherigen Protagonisten der Energiewende (Haushalte, 
Genossenschaften, Stadtwerke) nicht finanzierbar sind - das Aufspringen auf einen Zug ermöglicht werden, dessen Abfahrt vor über einem Jahrzehnt sie verpasst oder sogar zu verhindern versucht haben.

Der Konflikt um die künftige Gestalt einer Energieversorgung auf Grundlage erneuerbarer Energieträger, bei dem es letztlich darum geht, ob die Kontrolle über eine wichtige gesellschaftliche Infrastruktur wie bisher von einem Konglomerat großer Konzerne ausgeübt wird, ob die Spielräume für das lokale und nichtmonopolistische Kapital in diesem Bereich erweitert werden oder ob die Energieversorgung kapitalistischen Verwertungsinteressen gar ganz entzogen und demokratisiert wird, ist nur einer von vielen Konflikten, die in der Krise des fossilistischen Energiesystems ausgetragen werden.

In anderen Auseinandersetzungen, etwa jenen um die europäische Klimaund Energiepolitik, steht die prioritäre Förderung der erneuerbaren Energien und damit die Systemtransformation selbst zur Disposition. So setzt das 2014 beschlossene Klima- und Energiepaket der EU viel stärker als sein Vorgänger aus dem Jahr 2007 auf, Technologieneutralität“ bei der Bekämpfung des Klimawandels: $\mathrm{Ob}$ die - in ihrer Höhe selbst strittigen - europäischen Ziele zur Reduktion von Treibhausgasemissionen mittels des Ausbaus von erneuerbaren Energien erreicht werden oder über eine Renaissance der Atomkraft und durch neue, dank der carbon-captureand-storage-Technologie " $\mathrm{CO}_{2}$-arme“ Kohlekraftwerke, sollen die EU-Mitglieder selbst entscheiden dürfen. Dieser Kurswechsel der europäischen Energiepolitik ist der Tatsache geschuldet, dass es Staaten wie Polen, deren interne Kräfteverhältnisse stark von fossilistischen Akteuren dominiert werden, nicht zuletzt vor dem Hintergrund der Wirtschaftskrise gelingt, ihre Interessen zunehmend erfolgreich zu artikulieren (Geden/Fischer 2014).

Wird also der Übergang von einem System der fossilen zu einem der (dezentral zu erzeugenden) erneuerbaren Energien auf dem Stromsektor gerade bekämpft, so erlebt der Wärmesektor in Teilen der Welt, wie z.B. in den USA, derzeit einen Boom der nicht konventionellen Öl- und Gasförderung(Daniljuk 2015). Durch den Aufstieg der USA zum weltweit größten Ölproduzenten und durch den gegen Russland gerichteten Versuch, die Kooperation zwischen den USA und europäischen Staaten auf dem Gassektor zu intensivieren, erhält dieser Boom zusätzlich eine geopolitische Dimension. Eine sozial-ökologische Transformation des Energiesektors wird damit auch weiterhin mit erheblichen Beharrungskräften des fossilistischen Systems konfrontiert sein.

Im Verkehrssektor hat die Energiewende noch nicht einmal begonnen. Die private fossilistische Automobilität ist ungeachtet des Klimawandels nach wie vor das dominante Muster der Fortbewegung. In den Mittel- und Oberklassen aufstrebender Länder wie China breitet sie sich gerade mit Macht aus. Weltweit verzeichnen besonders ressourcen- und emissionsintensive Fahrzeuge derzeit unter allen neu zugelassenen $\mathrm{Pkw}$ die höchsten Zuwachsraten.

Im Vergleich zur Energiewende auf dem Stromsektor hat eine „Mobilitätswende“ deutlich weiterreichende Implikationen für die Lebensweise. Sie erfordert eine Transformation des beruflichen Alltags, der Freizeit- und Urlaubsgestaltung sowie der Stadtentwicklung. Wenn das Auto seine Zentralität verliert, hat das Auswirkungen auf symbolische Orientierungen (Vorstellung von Freiheit und Männlichkeit) sowie auf Klassen- und Geschlechterverhältnisse (Konversion einer männlich geprägten 
Branche wie der Automobilindustrie; Arbeitszeitverkürzung; Überwindung von suburbanen Siedlungsstrukturen, die fossilistische Formen der Fortbewegung ebenso perpetuieren wie asymmetrische $\mathrm{Ge}$ schlechterverhältnisse). Nicht zufällig beschränken sich bisherige verkehrspolitische Ansätze auf eine ökologische Modernisierung der fossilen Automobilität mittels des Elektromotors und der Beimischung von Agrartreibstoffen. Während die Politik der Elektroautomobilität ihren selbst gesetzten Zielen aber weit hinterher hinkt, bricht sich die Ausweitung der Agrartreibstoffproduktion am zunehmenden Widerstand in den Ländern des globalen Südens, teilweise auch des globalen Nordens (Dietz u.a. 2015).

Der Energiesektor ist damit auf vielfältige Weise umkämpft, politische Kräfteverhältnisse und deren Verschiebungen bilden sich in ihm ab. Die lange Geschichte der Umweltbewegungen ist ganz wesentlich eine Geschichte von Energiekämpfen. Die Krise gesellschaftlicher Naturverhältnisse wird an Energiefragen sichtbar; die Energiepolitik berührt ebenso Fragen der politischen Ökonomie wie solche der Aushandlung geopolitischer Interessen, der Dynamiken sozialer Bewegungen, der vorherrschenden Verbrauchsmuster und der geschlechtsspezifischen Rollenzuschreibungen.

Die Transformation des Energiesystems bietet auch Chancen für emanzipatorische Projekte. Mittlerweile hat sich eine lebhafte, sowohl aktivistische als auch wissenschaftliche Debatte entwickelt, die sich mit Energiefragen aus einer explizit kritischen Perspektive beschäftigt. Stichworte hierfür sind die Forderungen nach einer world-wide energy revolution (Abramsky 2010), nach energy justice (Bickerstaff et al. 2013), dem right to energy (Walker 2015) oder Energiedemokratie (Kunze/Becker 2015). Vor allem in Großbritannien spielt die Auseinandersetzung mit Energiearmut eine wichtige
Rolle in den Kämpfen um eine Neuausrichtung der Energieversorgung (Boardman 2010). In der Bundesrepublik stehen aktuell die Gründung von kommunalen Stadtwerken, Energiegenossenschaften oder energieautonomen Gemeinden für konkrete Vorhaben einer sozial-ökologischen Energieversorgung und zumindest teilweise für die Abkehr von neoliberalen Prämissen in der Steuerung von Infrastruktur (Becker u.a. 2015). Doch auch diese Tendenzen hin zu einer Dezentralisierung der Energieversorgung sind von Widersprüchen geprägt. So gehen Dezentralisierung und Demokratisierung keineswegs notwendigerweise Hand in Hand. Mancherorts wird etwa die Aufstellung von Windrädern gerade mit demokratiepolitischen Argumenten bekämpft, die auf die Verletzung der Interessen und Beteiligungsrechte der lokalen Bevölkerung verweisen.

In ersten Beitrag gibt Markus Wissen einen Überblick über aktuelle Entwicklungstendenzen des globalen Energieregimes. Auf der Basis vor allem der Szenarien der Internationalen Energieagentur (IE) argumentiert er, dass die These vom Höhepunkt der Ölförderung (peak oil) angesichts des Booms der nicht konventionellen fossilen Energieträger nicht mehr ohne Weiteres haltbar ist. Kritisch zu beurteilen sind zudem solche Analysen, die von einer Konsolidierung der US-Hegemonie auf dem Weg der Energiepolitik ausgehen. Auch wenn die USA in jüngerer Zeit zum weltweit größten Ölförderer aufgestiegen sind, wird ihre dominante Position durch das energiepolitische Agieren Chinas und durch Interessengegensätze zwischen den kapitalistischen Staaten des globalen Nordens beeinträchtigt. Die entscheidende energiepolitische Herausforderung liegt in der Überwindung des sozial ebenso wie ökologisch zerstörerischen (neo-)fossilistischen Energieregimes. Dazu reicht 
ein bloßes greening der kapitalistischen Produktionsweise nicht aus. Vielmehr müssen das Niveau des Energieverbrauchs ebenso wie die energetischen Produktionsverhältnisse grundlegend infrage gestellt und transformiert werden.

Tobias Haas beleuchtet die Verknüpfung der bundesdeutschen Energiepolitik mit Entwicklungen auf der europäischen Ebene. Die Konflikte zwischen dem grünen, auf erneuerbaren Energieträgern aufbauenden und dem grauen, auf fossilnuklearen Strukturen basierenden Projekt sind gerade auch dadurch geprägt, dass sie auf unterschiedlichen räumlichen Maßstabsebenen (scales) angesiedelt sind. Die Versuche, die Energiewende zu verlangsamen und eine weitere Dezentralisierung zu stoppen, wurden maßgeblich durch EU-Richtlinien, vor allem der Generaldirektion Wettbewerb, flankiert und vorangetrieben. Diese skalaren Verschiebungen von Kräfteverhältnissen zugunsten des grauen Projekts manifestierten sich in der Novellierung des deutschen EEG im Jahr 2014. Damit wird deutlich, dass energiepolitische Themen maßgeblich auch über die Frage der jeweils geeigneten Ebenen und deren Verschränkungen - der politics of scale - verhandelt werden.

Achim Brunnengräber und Felix Syrovatka untersuchen die unterschiedlichen Strategien der Anti-Atom-Bewegung bei der Endlagersuche für radioaktive Abfälle. Der Versuch, mit der Einsetzung der Endlager-Kommission 2014 VertreterInnen der Zivilgesellschaft einzubinden, stellte die Anti-Atom-Bewegung vor die Herausforderung, ihr Verhältnis zum Staat neu zu bestimmen. Innerhalb der Bewegung führte dies zu einer Polarisierung zwischen Akteuren, die sich an der Kommission beteiligten, und denjenigen, die eine Teilnahme ablehnen. Damit werden Fragen der Kooptation von sozialen Bewegungen und der Hegemonie in politischen Entscheidungsprozessen thematisiert.

Die Rolle sozialer Bewegungen diskutiert Hendrik Sander am Beispiel der Klimagerechtigkeitsbewegung in Deutschland. Diese Bewegung differenzierte sich nach der Mobilisierung zum internationalen Klimagipfel in Kopenhagen in einen „sozial-ökologischen“ und einen „radikalökologischen“ Flügel aus. Beide streben eine grundlegende Transformation des Energiesystems und der diesem zugrunde liegenden gesellschaftlichen Verhältnisse an. Während der sozial-ökologische Flügel Sander zufolge aber vor allem auf den Aufbau von Alternativen setzt, konzentriert sich der radikal-ökologische auf das Zurückdrängen der Energiekonzerne, die weiter auf Kohle als Energieträger setzen, und organisiert Aktionen wie die Energieund Klimacamps oder die Ende-GeländeKampagne. Damit versucht dieser Teil der Klimagerechtigkeitsbewegung, innerhalb des grünen Hegemonieprojekts die Kräfteverhältnisse hin zu kapitalismuskritischen Positionen zu verschieben. Sander zeigt, wie eine historisch-materialistische Analyseperspektive dazu beitragen kann, die Entwicklung, Möglichkeiten und Grenzen der Bewegung besser zu verstehen.

Die räumliche Dimension der Transformation des Energiesystems am Beispiel der Agrartreibstoff-Problematik steht im Mittelpunkt des Beitrags von Oliver Pye, Bettina Engels und Kristina Dietz. Die Nutzung von Agrartreibstoffen geht mit einer Verschiebung von gesellschaftlichen und internationalen Kräfteverhältnissen einher, die aus einer raumtheoretischen Perspektive besser begriffen werden kann. Hierbei sind die vier Dimensionen Netzwerke, Territorium, scale und place von besonderer Bedeutung. Dies zeigen die AutorInnen anhand von globalen Produktionsnetzwerken, Reterritorialisierungen 
durch Palmölplantagen, der Reskalierung von Finanzmärkten sowie lokalen Auseinandersetzungen um Flächennutzungen für die Agrartreibstoffindustrie. Dabei wird deutlich, wie sich neue soziale Disparitäten über die räumliche Restrukturierung der Energieversorgung herausbilden.

Der Einspruch von Ulrich Schachtschneider nimmt die aktuelle Novellierung des Erneuerbare-Energien-Gesetzes zum Anlass, die mit einer "Bürgerenergiewende“ bzw. einem „Green New Deal“verknüpften Erwartungen kritisch zu hinterfragen. So sind Investitionen in Anlagen erneuerbarer Energieträger für Haushalte mit niedrigen Einkommen nicht möglich, obwohl diese die Förderung solcher Investitionen über den Strompreis mit finanzieren. Demgegenüber ist ein sozio-ökonomischer Kulturwandel notwendig, der sich vom Paradigma stetigen Wachstums verabschiedet. Degrowth bedeutet dabei nicht nur weniger Energiebedarf, sondern auch die Abkehr von einer ,imperialen Lebensweise“. Die Debatte um die Widersprüche zwischen einem grünen Kapitalismus und einer grundlegenden sozial-ökologischen Transformation wird maßgeblich anhand von Energiefragen geführt werden müssen.

David Döll greift in seinem Einspruch die Nuit-Debout-Analyse von Felix Syrovatka in PROKLA 183 auf. Mit den Protesten in Frankreich, so Döll, haben die Anti-Austeritäts-Proteste ihren nördlichsten Punkt erreicht und die „Bewegung der Plätze" trifft auf Klassenkämpfe. Diese „Bündelung der Kämpfe“ (convergence des luttes) markiere eine neue Qualität, wobei gerade von der Organisationsform zu lernen sei, da sie bereits eine strategische Bündelung von Demokratie- und Klassenkämpfen antizipiert.

Außerhalb des Schwerpunkts würdigt Alex Demirović die vor 50 Jahren erschienene Negative Dialektik, eines der wichtigsten Werke von Theodor W. Adorno. Die Negative Dialektik zählte Adorno zu jenen Werken, die er ,in die Waagschale zu werfen habe“. Für Demirović ist die Schrift, die im Herbst 1966 erschien, eine Fortsetzung des Marxismus, „die Form, in der er sich selbst reflektiert", ein notwendiger Schritt nach den Erfahrungen des Nationalsozialismus, wenn die Kritische Theorie „ihren emanzipatorischen Gehalt bewahren und erneuern will.“

Ulf Kadritzke bereitet im ersten Teil seines Beitrags zum Begriff und Phänomen der Mittelklassen soziologische Analysen der Weimarer Republik auf, die, so seine These, weit mehr zu bieten haben, als die derzeit gegenwärtige gesellschaftspolitischen Ausdeutungen der Mitte, die den Klassenbegriff gerade los haben wollen und sich keine Rechenschaft darüber ablegen, „welche begrifflichen, theoretischen und damit auch politischen Vorentscheidungen in den Verfahren und empirischen Fragestellungen eingeschlossen sind“. Im zweiten Teil (in PROKLA 185) wird Kadritzke vor dem Hintergrund der ,Weimarer Soziologen" aktuellen Arbeiten auf den Zahn fühlen.

\section{Matthias Naumann und Markus Wissen (für die Redaktion) $* * *$}

Als Gastredakteur hat Matthias Naumann am Schwerpunkt dieses Heftes mitgewirkt. Die PROKLA-Redaktion dankt ihm ganz herzlich für seine Ideen und sein Engagement.

\section{Literatur}

Abramsky, Kolya (Hg.) (2010): Sparking a Worldwide Energy Revolution. Social Struggles in the Transition to a Post-Petrol World. Oakland. 
Becker, Sören/Beveridge, Ross/Naumann, Matthias (2015): Remunicipalization in German cities: contesting neo-liberalism and reimagining urban governance? In: Space and Polity 19(1): 76-90.

Bickerstaff, Karen/Walker, Gordon/Bulkeley, Harriet (Hg.) (2013): Energy Justice in a Changing Climate. Social equity and lowcarbon energy. London.

Boardman, Brenda (2010): Fixing Fuel Poverty. Challenges and Solutions. London.

Daniljuk, Malte (2015): America’s T-Strategy. Die US-Hegemonie und die Korrektur der US-Außen- und Energiepolitik. In: PROKLA 45(4): 529-544.

Dietz, Kristina/Engels, Bettina/Pye, Oliver/ Brunnengräber, Achim (Hg.) (2015): The Political Ecology of Agrofuels. Abindgdon.
Geden, Oliver/Fischer, Severin (2014): Moving Targets. Die Verhandlungen über die Energie- und Klimapolitik-Ziele der EU nach 2020. SWP-Studie. Berlin. URL: swpberlin.org, Zugriff: 11.7.2016.

Kunze, Conrad/Becker, Sören (2015): Wege der Energiedemokratie. Emanzipatorische Energiewenden in Europa. Stuttgart.

Umweltbundesamt (2015): Daten zur Umwelt 2015. Dessau. URL: umweltbundesamt.de, Zugriff: 11.7.2016.

Walker, Gordon (2015): The Right to Energy: Meaning, Specification and the Politics of Definition. In: L'Europe en Formation 378: 26-38.

\section{PROKLA 185 Ausnahmezustand - Sozialismus oder Barbarei? (Dezember 2016)}

Die politische Zeit hat sich in den vergangenen Jahren enorm beschleunigt. Die Ereignisse und Probleme lösen sich in immer schnellerem Rhythmus ab. Die kritische Analyse kommt ins Hintertreffen, sie hinkt der schnellen Abfolge, der Verschiebung der Herausforderungen und Prioritäten hinterher. Wir befinden uns an einem Punkt, an dem die Bemühung, den Entwicklungen nachzuspüren und Zusammenhänge zu erschließen, atemlos macht - und irgendwie vergeblich wirkt. Dieser „Ausnahmezustand“ bedarf selbst einer kritischen Analyse. Dabei ist mit „Ausnahmezustand“ nicht einfach eine Herrschaftspraxis jenseits rechtsstaatlicher Prinzipien gemeint, die es zunehmend auch gibt, sondern die zeitdiagnostische Feststellung: die Welt scheint aus den Fugen. Aber sind wir tatsächlich mit einer "Chaotisierung" gesellschaftlicher Verhältnisse konfrontiert? Oder sind nicht vielmehr Muster im Chaos zu erkennen? Und: Stehen die vielen beunruhigenden Ereignisse, die scheinbar nichts miteinander zu tun haben, nicht vielmehr doch in einem (welt-)politischen Zusammenhang, den eine Analyse herausstellen müsste? Was verarbeitet die zunehmend autoritär auftretende Staatsgewalt - allein eine politische Rechtsverschiebung oder viel eher eine Polarisierung gesellschaftlicher Verhältnisse? Warum fassen überkommene herkömmliche Begriffe - von „autoritärer (Wettbewerbs-)Etatismus“ über „Doppelstaat“ bis „Faschismus“ - analytisch nicht, was derzeit passiert? 